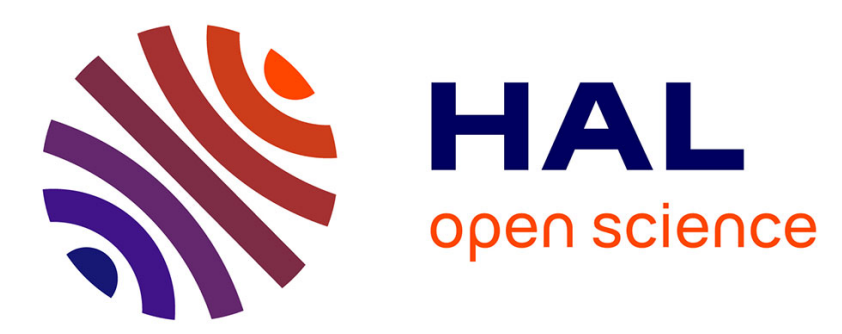

\title{
Dynamique récente des relations aux zones humides des paramos andins vénézuéliens : entre fonctionnalisme et mystique
}

Alexandra Angeliaume-Descamps, Frédérique Blot, David Leroy

\section{To cite this version:}

Alexandra Angeliaume-Descamps, Frédérique Blot, David Leroy. Dynamique récente des relations aux zones humides des paramos andins vénézuéliens: entre fonctionnalisme et mystique. Géocarrefour Revue de géographie de Lyon, 2013, 88 (4), pp.1-22. hal-01860372

HAL Id: hal-01860372

https://hal-univ-tlse2.archives-ouvertes.fr/hal-01860372

Submitted on 23 Aug 2018

HAL is a multi-disciplinary open access archive for the deposit and dissemination of scientific research documents, whether they are published or not. The documents may come from teaching and research institutions in France or abroad, or from public or private research centers.
L'archive ouverte pluridisciplinaire HAL, est destinée au dépôt et à la diffusion de documents scientifiques de niveau recherche, publiés ou non, émanant des établissements d'enseignement et de recherche français ou étrangers, des laboratoires publics ou privés. 


\title{
Géocarrefour
}

vol. $88 / 4 \mid 2013$

Nouveaux regards sur les zones humides

Articles

\section{Dynamique récente des relations aux zones humides des páramos andins vénézuéliens : entre fonctionnalisme et mystique}

Recent dynamics of the relations in the wet zones of the Venezuelan Andean paramos: between functionalism and mystic

\section{Alexandra ANGÉliaume-Descamps, FrÉdÉRIQue Blot AND DaVid LEROY}

p. $285-298$

\begin{abstract}
s
Français English

Cette contribution porte sur la dynamique récente des relations aux zones humides du páramo andin vénézuélien. Il s'agit de présenter quels sont les éléments déterminants de cette dynamique et quels sont les enjeux actuels relatifs aux divers acteurs. Ces espaces sont associés à des agriculteurs utilisant l'eau des zones humides pour l'irrigation et un parc national mis en place en 1952 prônant la mise sous cloche de l'hydrosystème. Suite à l'identification par certains des acteurs d'un problème de manque d'eau à l'aval, apparait une association croisant pratiques mystiques et actions de préservation des zones humides. Ce changement de configuration du système d'acteurs conduit à la requalification des zones humides.
\end{abstract}

This contribution concerns the recent dynamics of the relations in the wet zones of the Venezuelan Andean páramo. It is a question of presenting what are the determining elements of this dynamics and what are the current stakes relative to the diverse stakeholders Those spaces are associated to farmers using the water of the wetlands for the irrigation and a national park set up in 1952 pronant the putting under bell of the hydrosystem. Further to the identification by some of the actors of a problem of lack of water in the approval, appears an association crossing mystic practices and actions of conservation of the wetlands. This change of configuration of stakeholders system leads to the requalification of the wetlands. 


\section{Mots-clés}

représentations sociales, pratiques, régulation sociale, zones humides, biodiversité, espaces protégés, ressources en eau, Andes, páramos

\section{Keywords}

social representations, practices, social regulation, wetlands, biodiversity, protected spaces, water resources, The Andes, páramos

\section{Full text}

\section{Introduction}

Dans le contexte occidental d'un référentiel modernisateur et hygiéniste fondé sur des " mises en forme de la nature " tout particulièrement dans les pays occidentaux, les zones humides ont été, jusqu'à une période récente, plutôt perçues comme des espaces insalubres à aménager (Luginbuhl, 1992, p. 24-27 ; Ouzouf-Marignier, 1992, p. 80-82). Plus particulièrement il s'agit « d'assainir » par le drainage - terme très significatif des relations ${ }^{1}$ à ces espaces. Or il semble que, depuis les années 1970, l'on assiste à une dynamique des relations aux zones humides dont la convention RAMSAR (1971) constitue le signe le plus ostensible au niveau international. Cette dynamique est associée à un processus de valorisation qui repose sur une requalification des différentes composantes des hydrosystèmes et de leurs fonctions (Burnouf et al., 2004 ; Beck et al., 2007) - "fonctions relatives, au maintien de la biodiversité, à la qualité de l'eau, à la fixation du carbone, à la réduction des impacts des inondations " (Point, 2010). Les économistes proposent à ce titre d'évaluer les "services écosystémiques 》 qu'elles rendent aux sociétés (Salles, 2010).

2 Dans les Andes vénézuéliennes, et en particulier dans la Vallée du Haut Chama (figures 1 et 2), on assiste depuis la seconde moitié du xxe s. et plus particulièrement à la fin de la période à l'émergence de nouvelles relations aux zones humides des " páramos » andins ${ }^{2}$ (photo 1) directement liée à l'évolution du système d'acteurs associés à ces espaces. En effet, les connaissances produites notamment par les études écologiques menées par l'ICAE ${ }^{3}$ dès les années 1970, les besoins en eau croissants de l'agriculture irriguée développée dans les années 1960, où les politiques de protection des milieux (relevant d'INPARQUES4', avec la création du PNSN en 1952 et PNSC en 1989) ont fait émerger de nouvelles potentialités pour ces espaces et ont donc contribué à la construction de nouvelles relations au páramo. Ces relations ont aussi considérablement évolué depuis la création, en 1999, de l'association ACAR ${ }^{5}$ qui allie pratiques rituelles mystiques et actions de restauration dans le páramo. Aujourd'hui, l'ensemble des acteurs associés à la gestion des zones humides ${ }^{6}$ souligne l'importance de la préservation des zones humides pour garantir la ressource en eau. Les outils et les modalités d'actions sont certes différents et parfois sources de conflits, mais cet enjeu commun est clairement identifié par l'ensemble des acteurs.

Figure 1 : Les Andes vénézuéliennes, Sierra Nevada et Sierra Culata, parcs nationaux, lagunes et zones agricoles de la vallée du Haut Chama 


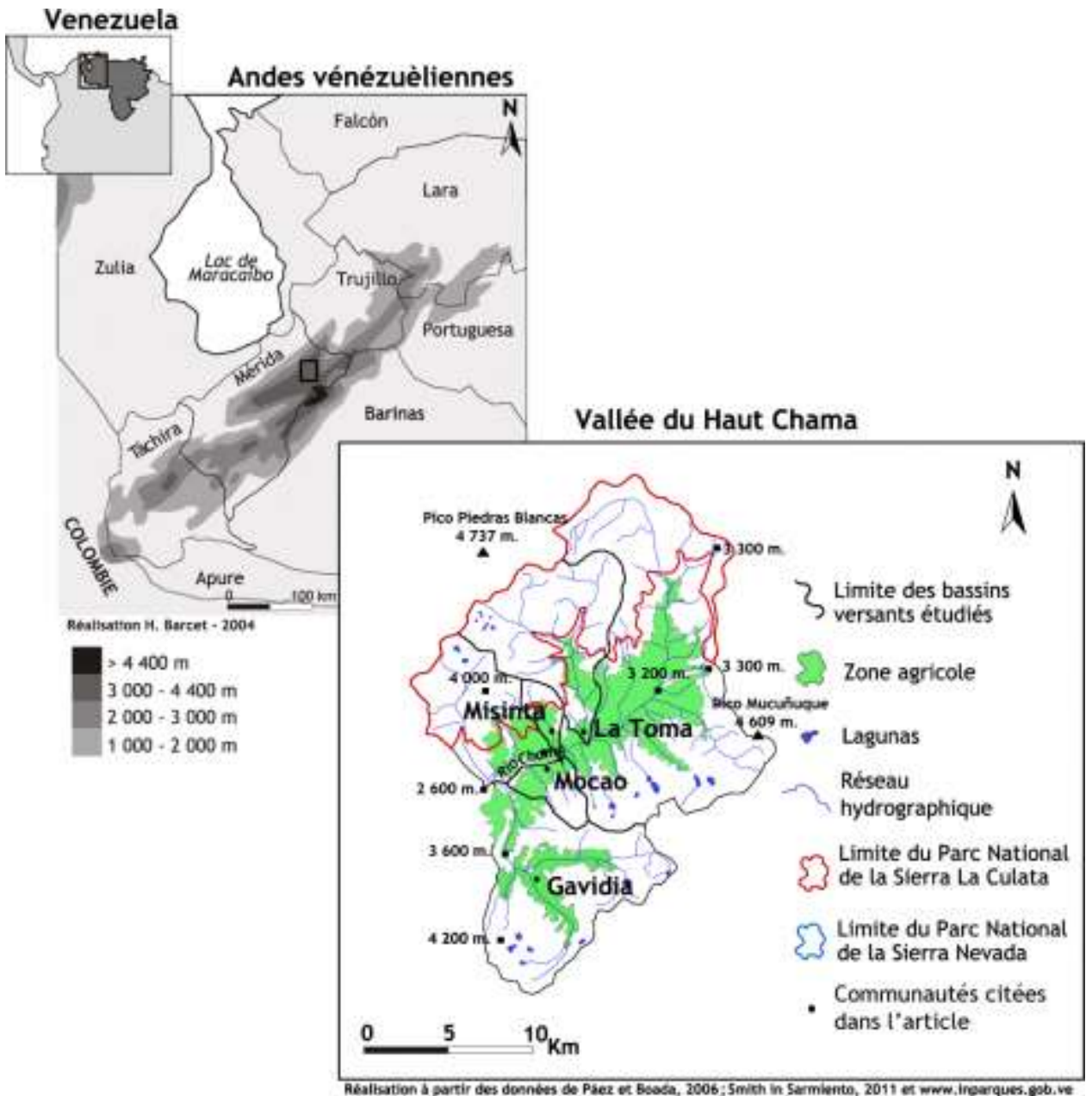

Figure 2 : Zoom sur une partie de la vallée du Haut Chama

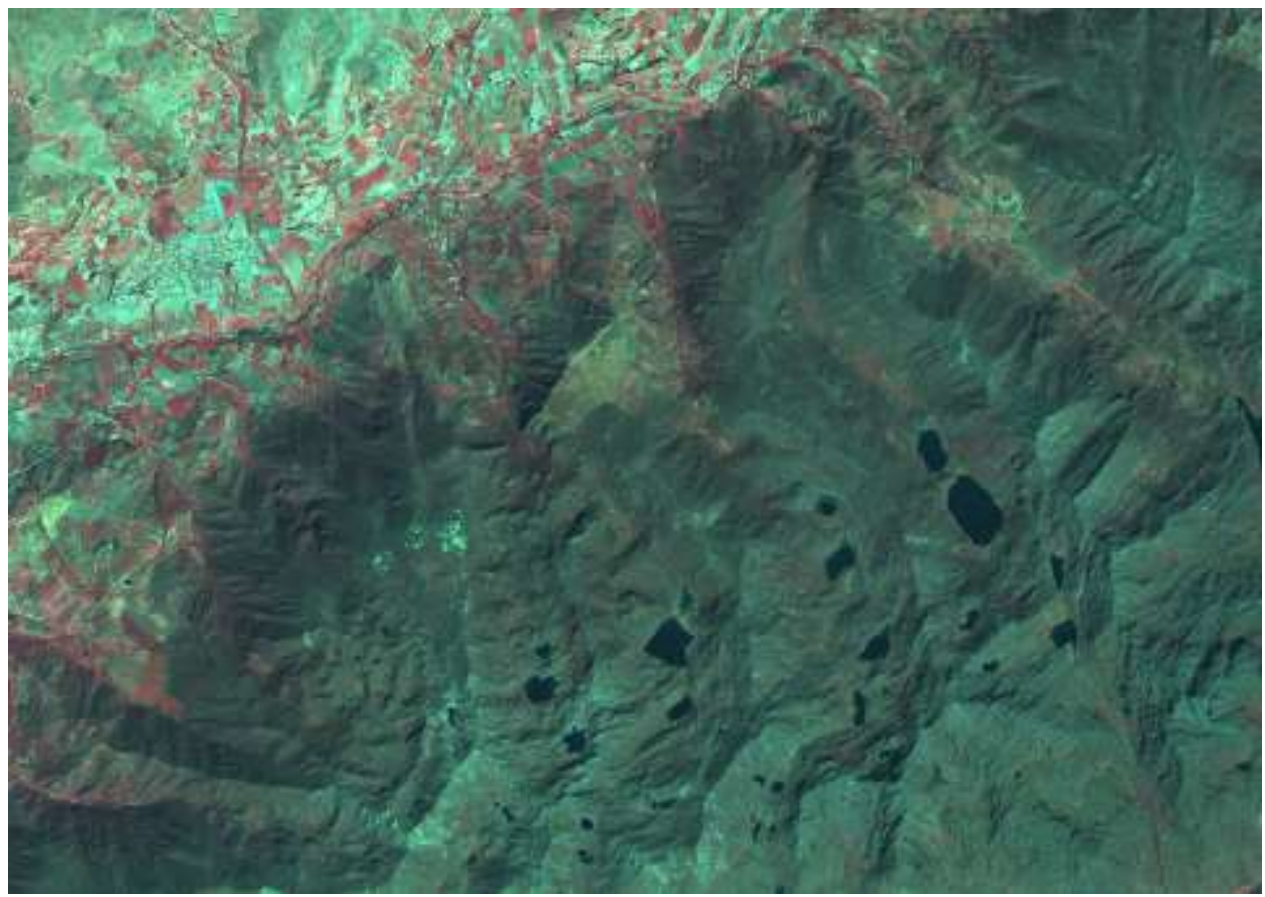

(Image satellite SPOT 2012 - composition colorée classique - programme ISIS SPOT PICS CNRS GEODE 5602 CNRS) : en rouge les zones agricoles de fond de vallée, en bleu les zones urbanisées et minérales, en noir les lagunas. C'est en marge de ces lagunas et dans les fonds de vallons que se concentrent les diverses zones humides.

Photo 1 : Les zones humides du páramo: 


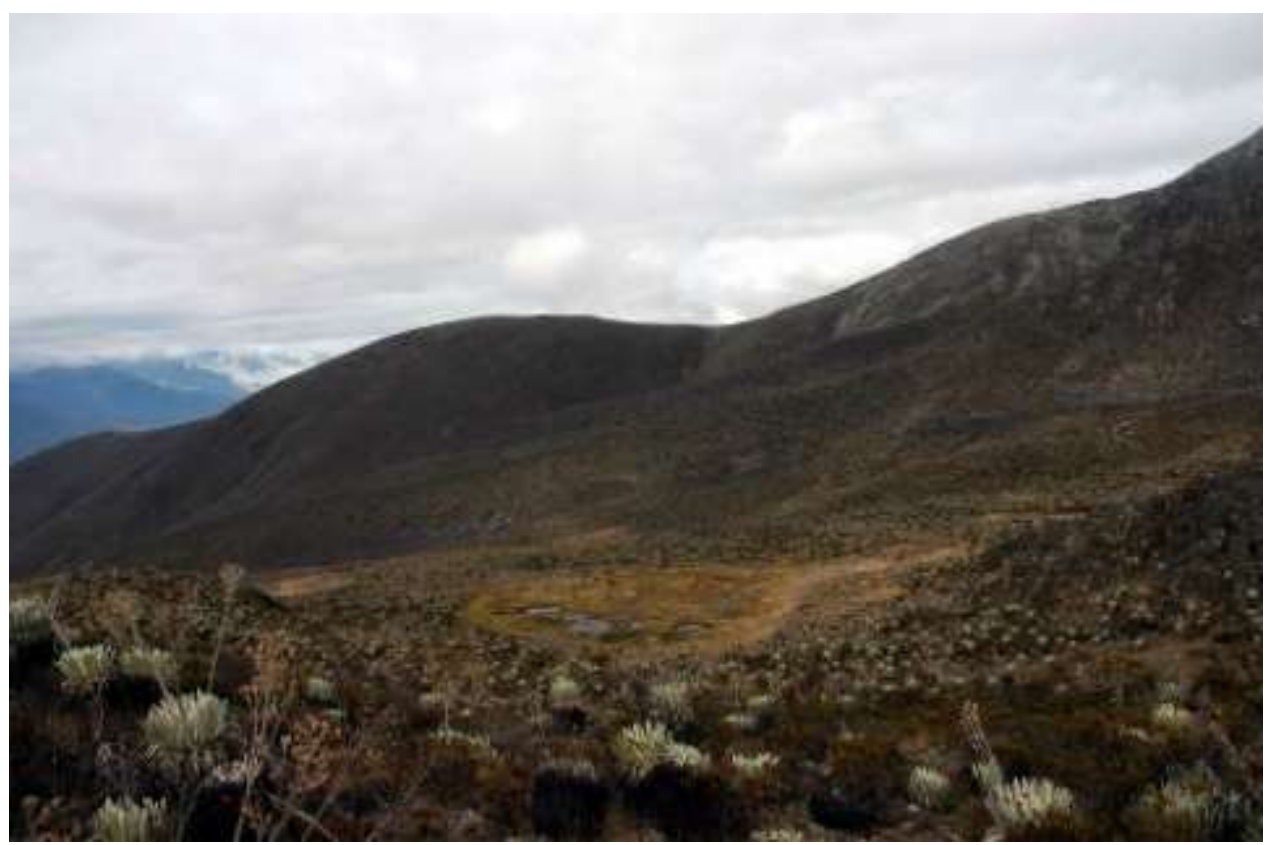

4

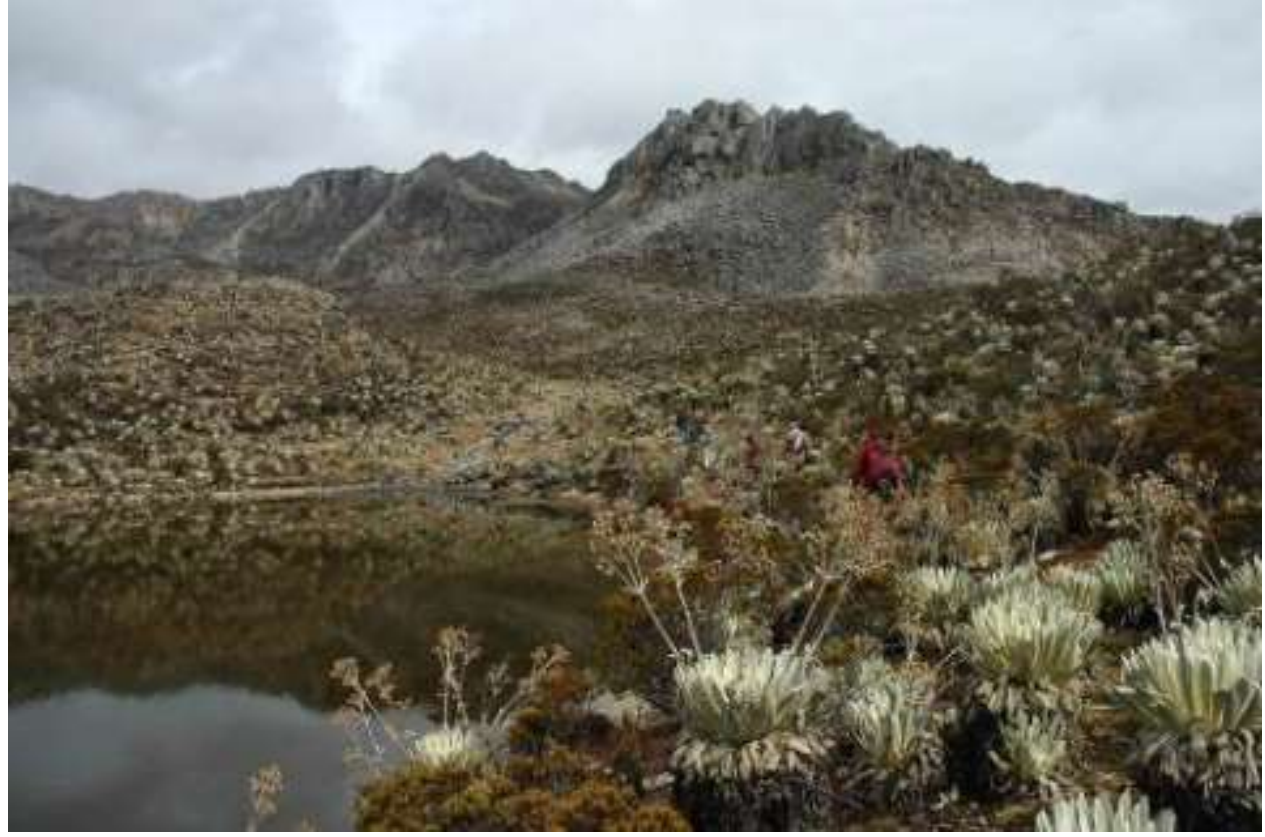

5

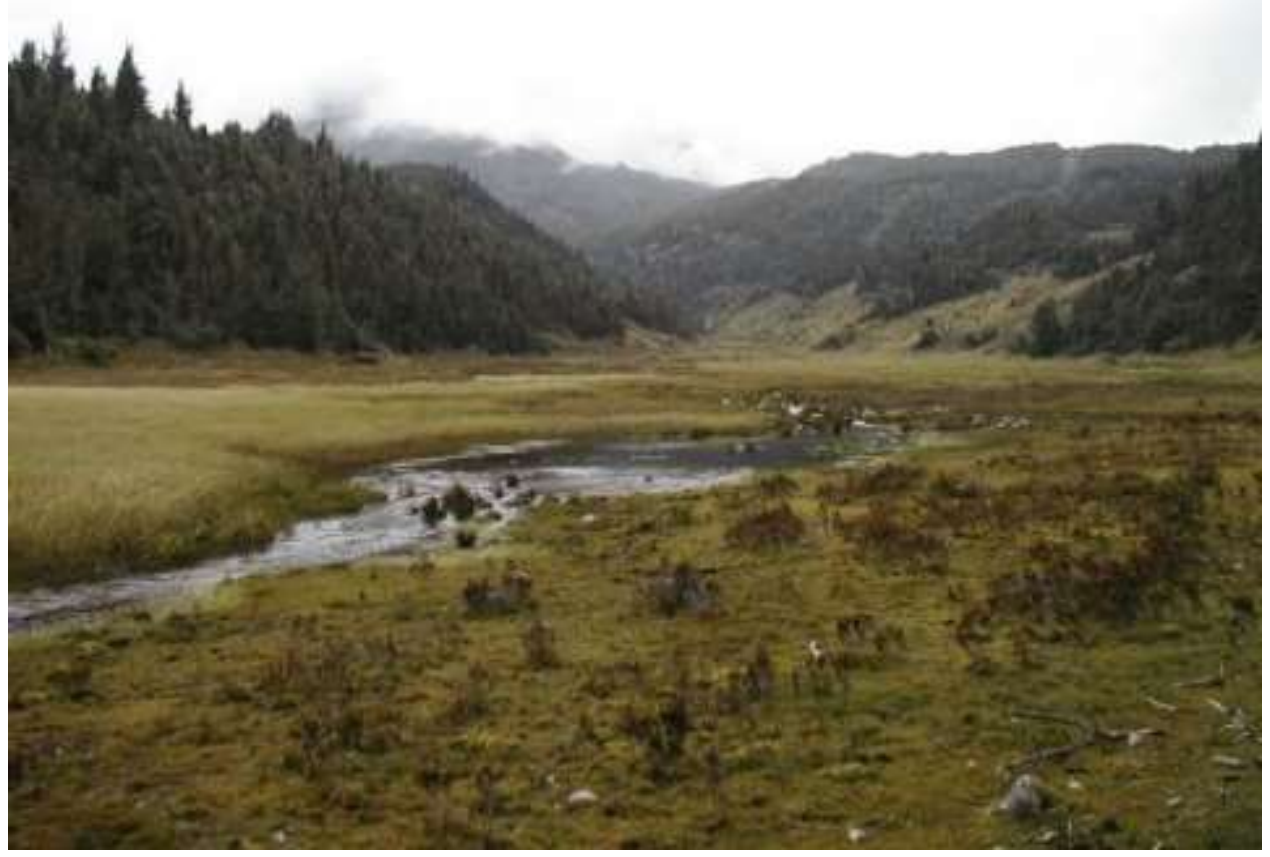

6 


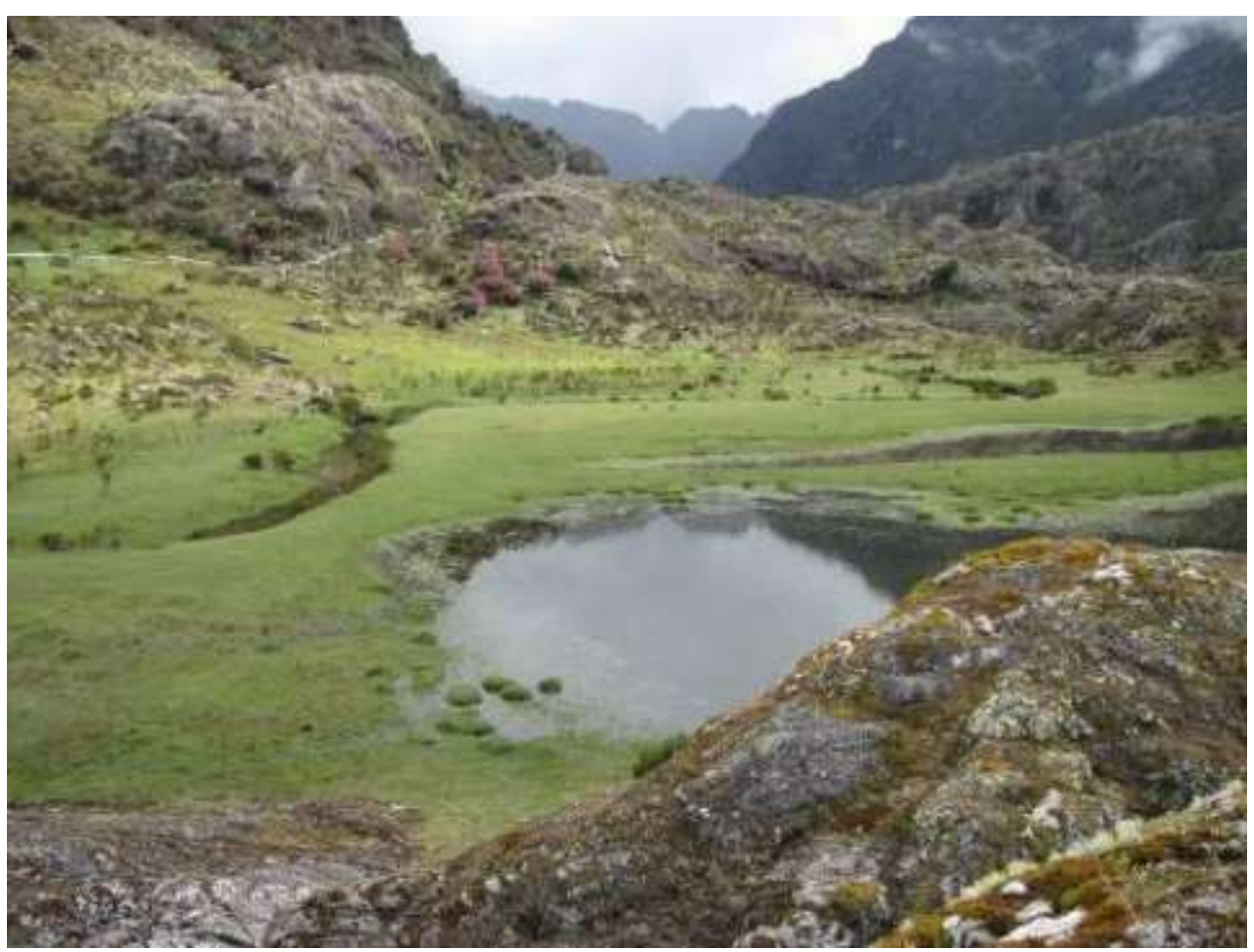

a) zones humides à l'amont de Llano de Hato, Sierra Culata (Blot, 2012) (b) lagunas de Llano de Hato, Sierra Culata (Blot, 2012), (c) amont de la laguna la Victoria, Sierra Culata (Angéliaume, 2007), (d) zone humide de fond de vallée et prise d'eau du périmètre irrigué de Mocao, Sierra Nevada (Angéliaume, 2010)

Cet article rend compte de l'évolution des représentations, des discours et des relations aux zones humides d'altitude récemment valorisées, et même honorées lors de cérémonies mystiques depuis le début des années 2000 suite à des épisodes de manque d'eau récurrents dans les fonds de vallées?.

8 Re-qualifier les zones humides, c'est redéfinir des caractéristiques - connaissances et donc une partie des relations aux zones humides. L'approche relationnelle telle que définie par Claude Raffestin (1980) est mobilisée ici pour appréhender ces dynamiques qu'il convient d'étudier pour en souligner les enjeux et mettre en avant les rapports de pouvoir entre différents acteurs. Au Venezuela, par exemple, depuis les années 1950, on assiste à la mise en évidence de nouveaux attributs et fonctions des zones humides d'altitude de la part de différents acteurs. On peut donc faire l'hypothèse que ces processus de requalification des zones humides engendrent une dynamique plus globale des relations, soit des pratiques et des processus de régulation existants. Mais aussi, en fonction des relations et des acteurs en présence, on assistera à des traductions locales pas forcément univoques compte-tenu des différentes rationalités, du niveau d'information et de la capacité à diffuser l'information des acteurs concernés.

9 Nous nous sommes donc intéressés à la façon dont les zones humides sont qualifiées - informations diffusées -, soit aux représentations qui sont construites et qui les caractérisent ainsi qu'aux pratiques à leur égard - mise en œuvre de principes considérés comme rationnels. Nous avons aussi étudié les processus de régulation qui se traduisent notamment dans la reconfiguration du système d'acteurs associé à la gestion de ces zones humides. Nous nous appuyions tout particulièrement sur des entretiens semi-directifs qualitatifs et sur l'observation participante mis en œuvre lors de missions de terrain entre 2010 et 2013.

10 La présentation se veut diachronique, puisque nous étudions la dynamique des relations aux zones humides. Nous présenterons dans un premier temps les relations plus anciennes, traditionnelles, aux zones humides du páramo vénézuélien à partir des travaux de l'anthropologue Pascale De Robert. Ensuite, nous étudierons les relations contemporaines et la façon dont elles se recomposent. 


\section{Conflits de relations aux zones humides dans les années 1950-1960}

\section{Pour les acteurs locaux les zones humides du páramo : un espace « risqué »}

Les zones humides du páramo sont avant tout, et historiquement, associées à des croyances incitant à l'éloignement et au respect : dans la culture andine vénézuélienne et dans toutes les Andes, les lacs ${ }^{8}$ sont très souvent la demeure d'êtres surnaturels, croyances très vives il y a encore peu de temps (De Robert, 2001, p. 233). L'eau et, en particulier, l'eau de l'« amont » occupe une place particulière, voire centrale, dans la mythologie andine vénézuélienne (De Robert, 2001, p. 73). Si certaines cascades abritent des êtres bienfaisants ou inoffensifs (anges, femmes, enfants), dont la présence est de bon augure, d'autres lieux, en raison de leur humidité, leur nébulosité, leurs brumes et brouillards sont considérés comme malsains : il s'agit des lacs du páramo et de leurs cortèges de zones humides. Le páramo est un espace exclusivement masculin où on ne se rend pas seul, un lieu qui fait l'objet d'un voyage initiatique pour tout petit garçon afin de découvrir les plantes, retrouver les traces du troupeau et, surtout, reconnaître les manifestations des cheses, les maîtres du páramo, et respecter la montagne (De Robert, 2001, p. 231).

Que l'on ait un « droit de páramo ${ }^{9}$ » ou pas (De Robert, 2001, p. 230), cet espace est le lieu des cheses : les hommes n'y sont plus maitres. Les cheses sont particulièrement attachés aux lacs puisqu'il s'agit de leurs demeures. Ils manipulent les éléments du climat, lorsqu'ils se mettent en colère, pouvant créer une tempête (où le marcheur peut disparaitre) ou ils peuvent offrir une éclaircie permettant de retrouver le taureau égaré depuis longtemps (De Robert, 2001, p. 231). Dangereux et puissants, n'ayant pas d'enfants, les cheses s'entourent d'enfants volés aux humains et d'animaux du bétail qu'ils entraînent au fond des lacs, les gens n'en reviennent pas, ils demeurent prisonniers. "Pour tenter d'amadouer cet espace insoumis au caractère ténébreux, les paysans adoptent des comportements particuliers à chacun de leur passage dans le páramo. Il faut d'abord éviter de troubler la quiétude de la montagne avec ses cris ou le bruit d'un éboulement, il convient de manifester de la crainte et surtout du « respect » en gardant en particulier ses distances avec les lacs où l'on ne pêche pas, en faisant régulièrement des offrandes d'alcool fort (miche) et de pâte de tabac (chimô) très prisées des cheses. » (De Robert, 2001, p. 232). Faute de quoi, confondant le monde des humains à celui des cheses, les prisonniers perdent la mémoire, la notion du temps et de la réalité. Pascale De Robert décrit le páramo comme un espace de confusion et de mélange qui n'obéit pas à l'ordre que l'on reconnait dans le territoire des hommes (De Robert, 2001, p. 235), c'est le domaine des génies mystérieux qui sont à la fois surnaturels et intégrés à la nature. Le páramo est un espace magique que personne ne peut prétendre contrôler. Il fallait alors se munir d'offrandes pour pouvoir espérer échapper à ces êtres redoutés.

Ainsi comme l'a largement décrit Pascale De Robert (2001, p. 221), le páramo est associé depuis l'époque coloniale à des terres désolées et incompatibles avec l'établissement d'humains, " un lieu marginal, domaine des êtres surnaturels qui habitent des lacs et des rochers " (De Robert, 2001, p. 217). Les agriculteurs ne s'y déplaçaient que très rarement pour récupérer le bétail lors de la saison sèche (De Robert, 2001, p. 226). Il s'agissait donc d'un espace risqué, peu valorisé par la population rurale.

\section{Mise en mouvement du pâturage : point de départ de la dynamique}


Bien que cela puisse paraître paradoxal, ces espaces étaient toutefois de longues dates utilisés comme lieux de pâturage pour les animaux. Couverts de pelouses denses, d'une végétation abondante et appétante pour le bétail, ils constituaient un lieu de pâturage saisonnier. Cette pratique a d'ailleurs été favorisée par les colons espagnols ${ }^{10}$ qui avaient introduit un droit d'usage nouveau : le derecho de páramo. L'activité de ganaderia a été développée par les espagnols : les animaux changeaient alors d'étage agricole au grès des cycles culturaux. Ainsi, pendant les périodes de jachère (descanso) ou d'interculture (barbecho), les animaux - ovins, bovins et équidés - rejoignaient les parcelles qu'ils fertilisaient de leurs déjections dans les vallées, puis pendant les périodes de culture, ils rejoignaient le páramo (à l'exception des vaches laitières qui demeuraient dans les pâturages des fonds de vallées près des fermes). Dans le páramo, les bêtes se regroupaient autour des zones humides : situées sur les replats de versant ou aux fonds des vallons, elles fournissaient une végétation dense (graminées) et régulière qui attirait le bétail et des points d'eau.

A partir des années 1960 dans le cadre de programmes de développement locaux (Programa Subsidio conservacionista 1962-1974 et Programa Valles Altos 1974-1988) visant à remédier à un fort exode rural et une extrême pauvreté, sont mis en place des systèmes agricoles fondés sur l'irrigation et prélevant l'eau dans ces espaces d'altitude. Les enjeux autour de cette nouvelle activité agricole sont forts : outre l'approvisionnement en produits maraîchers de nombreux centres urbains, elles assurent une très nette amélioration des conditions de vie ${ }^{11}$.

A l'aval, les animaux sont exclus des parcelles consacrées exclusivement au maraîchage et relégués dans le páramo (drainage des fonds de vallée pour mise en culture, disparition du pâturage saisonnier ou de la jachère pâturée). Ce cloisonnement spatial des activités renforce les conflits avec le PNSN, implanté depuis $1952^{12}$. De fait, les techniciens du parc entretiennent de longue date des relations conflictuelles avec les agriculteurs autour de la question du pâturage dans le páramo et de la pratique du brûlis pour renouveler les herbages des zones humides, de la remontée de la frontière agricole dans certaines zones du parc, de l'amplification des prises d'eau, etc. (Angéliaume-Descamps, 2010). Ces conflits atteignent leur apogée en 1992 avec la manifestation des agriculteurs ( "marcha de los bueyes ») qui obtiennent une révision du zonage en leur faveur, mais les relations restent tendues. Afin de réaliser son objectif de préservation du réservoir hydrologique, le parc a engagé une procédure de classification de la Vallée du Haut Chama en zone RAMSAR. Il espère ainsi mettre fin au droit de páramo, qualifié de «derecho negativo » pour évoquer les impacts négatifs du piétinement du bétail qui endommage la végétation, compresse les sols et perturbe les fonctions de rétention en eau.

17 Le páramo, et plus particulièrement ses zones humides, constitue donc un espace à fort enjeux pour les communautés rurales et le parc national jusqu'au milieu des années 1990. Ces enjeux sont, avant tout, relatifs à la façon dont les acteurs en jeu considèrent le fonctionnement des zones humides, mais aussi fonction de leurs pratiques en interaction avec ces espaces. Il apparaît aussi ici que la qualification de ces espaces est un indicateur des régulations à l'œuvre - rapport de pouvoir légitimant les pratiques de tel ou tel groupe d'acteurs - dans la construction des relations aux zones humides. Nous avons ainsi étudié les représentations des zones humides des principaux acteurs du Haut Chama.

\section{Les zones humides : représentations différentes mais compatibles}

\section{Les zones humides entre biodiversité et paysage}

Pour les instances du parc, les zones humides du páramo représentent avant tout un enjeu important en termes de biodiversité. Les communautés végétales sont composées 
d'espèces très distinctes de la végétation des différentes formations des páramos andino et altiandino adjacents. Même si ces zones humides représentent une superficie très restreinte, elles concentrent une grande quantité d'espèces végétales spécifiques ${ }^{13}$. Dans les tourbières présentes dans ces complexes humides, par exemple, on rencontre des espèces végétales endémiques et menacées telles que Uncinia meridensis, Carex collumanthus, Thriniochloa andina, Poa mucuchachensis, P. boxiana, Festuca fragilia, F. elviae, $F$. coromotensis ou Coespeleia moritziana. Ces écosystèmes présentent aussi une importance particulière pour les oiseaux migrateurs : ce complexe humide est ainsi considéré comme faisant partie des aires de conservation des oiseaux des Andes tropicales ; il appartient, en particulier, au corridor de conservation nord-andin du Venezuela des oiseaux migrateurs continentaux et locaux, tels que le pato de torrentes (Merganetta armata) et le pato serrano (Anas flavirostris) (INPARQUES, 2007). Les zones humides constituent donc un patrimoine naturel que l'on préserve pour sa faune et sa flore emblématique et/ou endémique.

Cette dimension écosystèmique est également reconnue et co-construite par les écologues de l'Université des Andes du Vénézuela (ULA) qui ont commencé à travailler sur le páramo au milieu des années 1970, peu de temps après la création (1969) du Centro de Investigaciones Ecológicas de Los Andes, Tropicales, CIELAT, qui deviendra en 1999, el Instituto de Ciencias Ambientales y Ecológicas, ICAE. Au cours des années 1980, ces écologues multiplient les études sur ces espaces de montagne, aux mêmes titres que sur les autres écosystèmes de la montagne tropicale comme la selva nublada, identifiant et inventoriant leur biodiversité spécifique. Les premiers travaux d'ICAE ${ }^{14}$, comme ceux de Maximina Monasterio (1980), constituent des références et correspondent à une approche descriptive de l'écosystème páramo. Ils présentent une description générale du páramo comme région naturelle, ses caractéristiques environnementales à plusieurs échelles, la description des principales formations végétales et une analyse de l'histoire de l'occupation humaine (Llambí, Sarmiento et Rada, 2013).

20 Enfin, la dimension paysagère de ces espaces est de plus en plus valorisée dans le contexte de l'importance croissante du secteur touristique dans les Andes de façon générale. Ces espaces d'altitude et les páramos andins sont l'objet d'une valorisation croissante qui en fait des lieux très prisés par les touristes vénézuéliens, mais aussi étrangers. A ce titre, il n'est pas rare de trouver des groupes de Hollandais, d'Allemands ou de Suisses qui sillonnent les sentiers éloignés de ces plus hautes terres, pratiques touristiques qui semblent acceptées par le parc.

\section{Les zones humides, des sources d'eau}

Au-delà de la dimension écosystémique et paysagère de ces zones humides, c'est surtout le rôle de réservoir hydrologique qui va concentrer les intérêts des divers acteurs, et ceci dès les années 1960 .

Ces étages andin et alti-andin forment, en effet, un lieu de captation des eaux et de formation de multiples sources qui alimentent un abondant et important réseau hydrographique, soit directement par ruissellement superficiel vers les quebradas ${ }^{15}$ et ríos $^{16}$, soit en transitant par d'autres zones humides. Ces diverses zones humides constituent des compartiments fondamentaux de réserves et de sources d'eau. Les sols profonds, la matière organique abondante et le microrelief qui les caractérisent en font de véritables éponges ou réservoirs temporels qui absorbent l'eau, en ralentissent la circulation, puis la libèrent à nouveau lentement pendant la saison sèche. Les sols peuvent retenir jusqu'à trois fois leur poids sec en eau, en relation avec leur forte teneur en matière organique, teneur favorisée par une faible activité de la microfaune minéralisatrice liée aux faibles températures. Un autre élément est essentiel à cette fonction hydrologique, aussi lié au climat : l'évaporation et l'évapotranspiration sont faibles et donc le bilan hydrique est largement excédentaire. Pourtant, par faute de moyens techniques et économiques, le rôle des zones humides dans le stockage et la régulation des flux hydriques a pour le moment été très peu étudié par les écologues. Une seule étude, en 2010, traite du rôle hydrologique du páramo et de ses zones 
humides (Rodriguez, 2010). Elle met en exergue l'importance de ces unités pour l'alimentation en eau des communautés de l'aval pendant les deux premiers mois de la saison sèche (Rodriguez, 2010).

Les techniciens du parc, eux aussi, accordent une attention particulière au rôle hydrologique de ces zones humides. La notion de système est ici centrale. En effet, les zones humides alto-andines ne sont pas appréhendées comme des points d'eaux isolées, mais, au contraire, comme des complexes ou systèmes de zones humides, qui prennent en considération l'ensemble des bassins hydrographiques récepteurs. On protège donc ces espaces car ils « produisent » de l'eau utilisée pour la consommation humaine et la production hydroélectrique, mais, aussi pour que « cette ressource soit utilisée de façon durable, pour assurer sa perpétuité et sa conservation à travers le temps » (Ingénieur 1, INPARQUES, 2011).

24 Enfin, le rôle du páramo comme réservoir naturel en eau a toujours été admis par les communautés de la vallée du Haut Chama (le Chama est le cours d'eau qui draine cette partie du sillon intra-andin). C'est d'ailleurs pour cette raison qu'elles ont développé, ces dernières décennies, de nombreuses infrastructures d'irrigation dans un processus de valorisation de ressources en eau de ces hydrosystèmes. Toutefois, bien que ce rôle hydrologique ne soit pas remis en question, l'importance des zones humides dans le stockage et la régulation de l'eau, était, il y encore peu d'années, très mal connue, voire ignorée des agriculteurs. Cette méconnaissance du fonctionnement de cet hydrosystème et, en particulier, du fonctionnement de ces zones humides, était avant tout liée à la méfiance de la population locale à l'égard de ces plus hautes terres.

A partir de 1999, une importante sécheresse et le manque d'eau orientent les regards de ces communautés vers les zones humides du páramo sous l'impulsion de Ligia Parra, coordinatrice de l'association ACAR $^{17}$ : il s'agit d'une étape clé dans l'usage et la gestion des zones humides et de la gestion de l'eau. Le fait que les agriculteurs et les acteurs locaux regardent à l'amont pour comprendre l'évolution hydrologique à l'aval souligne l'émergence d'une représentation hydrosystémique des zones humides.

"En 1999 [...] les sources qui donnent vie au bassin versant de Misintá étaient pratiquement toutes sèches, elles étaient toutes mortes. Nous avons eu l'idée de monter là-haut pour voir pourquoi il n'y avait plus d'eau. Quand nous sommes montés en haut, nous nous sommes rendus compte que les zones humides étaient sèches " Ligia Parra, Misintá, responsable de l'association ACAR, 2012.

Ainsi, c'est à partir de ce constat de dégradation et d'assèchement des zones humides que l'association ACAR est créée. Elle s'associe aux comités d'irrigation et aux agriculteurs afin d'œuvrer pour la préservation des zones humides, avec des actions de restauration, ou la mise en place de périmètres de protection. Les zones humides sont alors appréhendées comme des sources d'eau. Cette association a eu des débuts particulièrement difficiles. Les représentations systémiques qu'elle médiatise contribuent effectivement à proposer des actions qui apparaissaient opposées à la pratique de l'élevage en altitude, remettant alors en cause le « droit de páramo » de certains éleveurs. C'est à force de prises de parole dans les comités de riego ${ }^{18}$ que Ligia Parra a contribué à valider des représentations systémiques des bassins-versants et à faire mettre en ouvre par les agriculteurs eux-mêmes des projets de protection des zones humides dans la vallée du Haut Chama.

28 ACAR véhicule aussi des représentations particulières des zones humides. C'est, en effet, à son initiative que sont organisées des cérémonies en l'honneur de la " madre naturaleza ». Nous avons pu observer et participer à l'une de ces cérémonies. Les zones humides sont alors tour à tour " création ", " don de Dieu ", " mère nature ». La relation est aussi fonctionnaliste, les zones humides sont considérées pour ce qu'elles « font » pour les sociétés locales. Ici, on valorise leurs caractéristiques nourricières grâce à l'eau qu'elles véhiculent et qui permet de produire les aliments et l'eau « essentiels à la vie ». Par conséquent, les membres d'ACAR affichent leur « respect » envers la " madre naturaleza » et souhaitent la "vénérer », la " protéger », en « prendre soin » et l'« aimer » (Ligia Parra, Misintá, responsable de l'association ACAR, entretiens 2011-2012). 
Figure 3 : Evolution temporelle des représentations et des enjeux des divers acteurs autour des zones humides des páramos andins de la vallée du Haut Chama

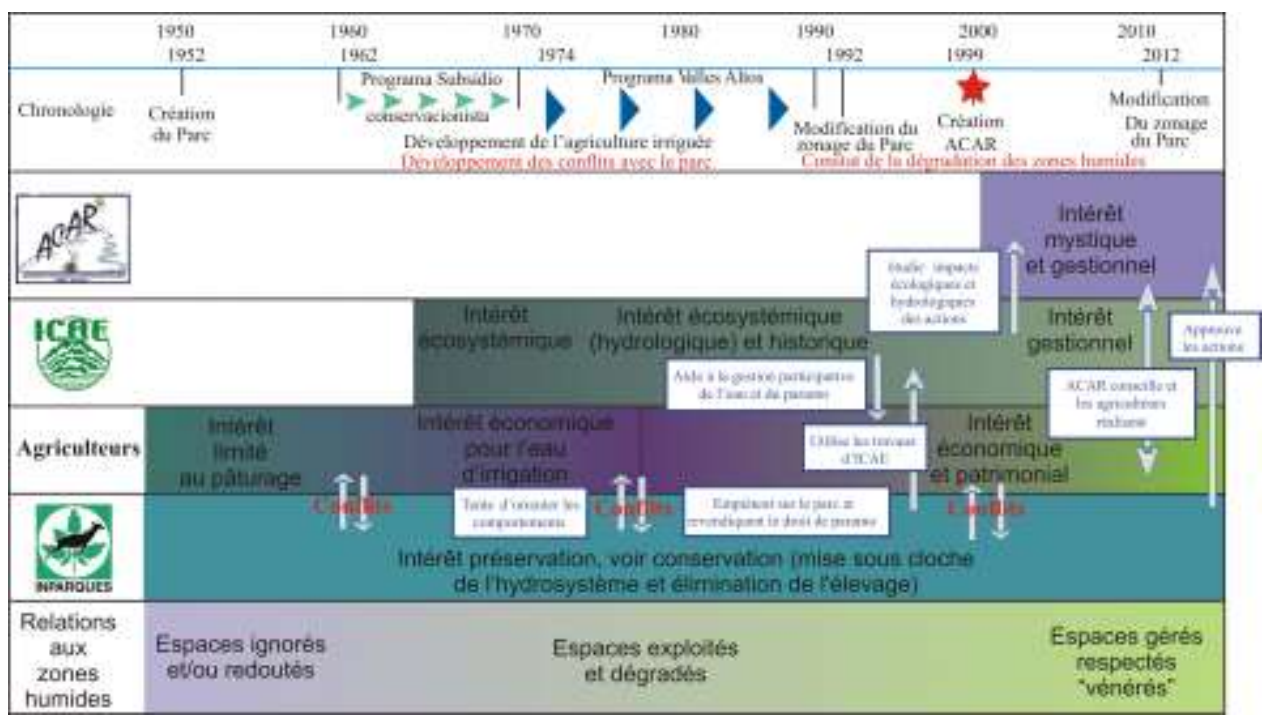

\section{Les nouvelles relations au cœur de processus de régulation}

Les représentations précédentes renvoient à des pratiques différentes et concourent à des relations différentes : des relations fondées sur l'étude et la gestion de la biodiversité de ces espaces, des relations fondées sur les usages de l'eau qui s'écoule de ces zones humides vers l'aval, des relations mystiques. Pourtant, dans l'ensemble, la dynamique de construction de ces différentes relations aux zones humides conduit à identifier un problème commun - l'altération des zones humides par le piétinement et à définir un objectif de gestion commun - la protection des zones humides.

\section{Le piétinement des zones humides en question?}

Pour les agriculteurs, si le páramo constituait autrefois un excellent lieu de pâturage pour les troupeaux, notamment pendant toute la saison pluvieuse, sa présence permanente a entraîné, ces dernières années, une diminution importante du fourrage. Ainsi, "l'élevage n'a pas d'autre endroit où pâturer que dans les sources, les zones humides » là où le fourrage est beaucoup plus dense. Aujourd'hui, la dégradation des zones humides par la présence du bétail est un diagnostic partagé par l'ensemble des communautés de la vallée. La sensibilisation assurée par les membres de l'association ACAR a conduit la plupart des agriculteurs à expliquer, avec plus ou moins d'aisance, comment le bétail altère les points d'émergence de l'eau par piétinement.

\footnotetext{
«Une des choses qui a beaucoup influencé la diminution de l'eau, c'est l'élevage, l'élevage qui était laissé comme ça dans le páramo, là où apparaissent les sources, l'eau est plus fraîche et plus verte. L'élevage, c'est ce qui enfonce à travers les piétinements. » Dionsio Espinoza, 65 ans, agriculteur de Misintá, 2011.
}

Pour certains ingénieurs du parc le diagnostic est identique :

\footnotetext{
« Cette concentration [d'animaux] est à l'origine d'un effet de compression, le sol de ces zones humedales, turberas, ciénagas, pantanos, céspedes ${ }^{19}$ se comprime, cela diminue bien sûr leur capacité de rétention en eau. » Ingénieur 1, INPARQUES, 2010.
}

Les écologues, eux aussi, mettent en avant le rôle du bétail dans la diminution significative de 30 à $40 \%^{20}$ de la biomasse végétale, de la diversité végétale et de l'abondance relative d'un grand nombre d'espèces (Molinillo et Monasterio, 2003 ; Lermis et al., 2007 ; Llambí et al, 2013). C'est en effet au cours de recherches engagées 
au début des années 1990 qu'ICAE a orienté une partie de sa réflexion sur la relation entre l'élevage et les changements de végétation, en particulier dans le complexe des zones humides du páramo (Molinillo 1992 ; Molinillo, Monasterio 1997), et que, finalement, l'activité pastorale dans le páramo a commencé à être identifiée comme problématique.

Les différentes représentations de ces zones humides renvoient ici à un même objectif : il faut préserver les zones humides du piétinement pour favoriser leur rôle de zone tampon hydrologique, tout comme pour favoriser la biodiversité spécifique qui assure le rôle de zone tampon.

Toutefois, à ce jour, aucune étude scientifique n'a encore mis en évidence l'impact réel que représentent les actions de protection des zones humides sur les conditions d'écoulement hydrique. Seule une étude s'est intéressée en 2010 à la régénération de la végétation, à la diminution du compactage des sols, ainsi qu'à l'augmentation de la capacité maximale de rétention de l'eau à l'intérieur des périmètres protégés (Valero, 2010). Mais, comme nous l'a affirmé un membre d'ICAE, il est encore particulièrement difficile de tirer des conclusions sur l'impact des zones de protection sur la ressource eau, notamment à cause d'une concordance de ces actions avec une augmentation des précipitations sur la même période.

35 "Si on regarde la séquence temporelle des précipitations de Mucuchies, les années à partir de 2004 ont eu des moyennes de précipitation au-dessus de la normale, c'est donc logique qu'il $y$ ait plus d'eau dans la quebrada, mais savoir si cela est directement en relation avec l'exclusion de l'élevage, cela est très difficile. »Chercheur d'ICAE, 2011.

Certains agents du parc font un autre diagnostic.

"Le bétail ne va pas affecter les zones humides. Même si on enferme dans une zone humide 30 vaches... la dynamique naturelle de ces micro-écosystèmes permet qu'ils récupèrent plus rapidement que l'affectation produite pas ces vaches... » Ingénieur 2, INPARQUES, 2011.

38 Le rôle du bétail dans la dégradation des zones humides est donc en débat au sein même d'INPARQUES. Or les objectifs de gestion associés à ces différents diagnostics sont opposés : dans un cas, il s'agit d'exclure le bétail du páramo, dans l'autre, il faut conserver les pratiques pastorales, activité ancestrale garantissant un revenu secondaire (fromage, viande des animaux en cas de mauvaise récolte, même si les vaches sont généralement maigres).

39 "Moij’y vois une utilité surtout ancestrale, d'un point de vue du paysan. Une utilité culturelle, ancestrale et même économique. Le bétail qu'a le paysan dans le páramo est une sorte de garantie d'économies futures. [...] Comment peut-on obliger le producteur du páramo à ne pas avoir de bétail et à ne pas pouvoir faire de fromage? Il faudrait alors qu'il achète le lait au marché pour faire son fromage, alors que celuici vient de la campagne, ce n'est pas logique. Ainsi, d'un point de vue culturel, je revendique la présence du bétail, mais en prenant en compte certains critères de conservations des zones humides. » Ingénieur 2, INPARQUES, 2011.

\section{Clôturer, c'est s'approprier les zones humides}

Certains agriculteurs qui peuvent partager le diagnostic conduisant à la nécessité de protéger les zones humides rejettent pourtant cette solution et notamment les aides du parc - en particulier la fourniture de piquets. C'est le cas de la communauté de Mocao qui ne souhaite pas légitimer les principes rationnels de gestion de ces espaces du parc.

"INPARQUES nous a offert des piquets pour clôturer les sources, mais nous ne pouvons pas permettre cela, parce que les sources n'appartiennent pas à INPARQUES, les sources appartiennent à toute la communauté. Si nous permettons qu'INPARQUES nous donne des piquets, à n'importe quel moment ils peuvent dire que les sources sont à INPARQUES et que nous ne pouvons pas les utiliser pour l'irrigation, ensuite il faudrait s'adapter à leurs propres normes » Président du Comité de Riego de Mocao, 2011. 
42 Des problèmes, persistent dans certaines communautés quant à la pratique du « droit du páramo »,. C'est notamment le cas de la communauté de la Toma où certains agriculteurs refusent d'exclure le bétail des zones humides.

43 "Le problème, c'est que beaucoup de personnes ont des "droits dans le páramo », et, comme beaucoup ont des animaux, ils y vont et personne ne les surveille, ils enlèvent les clôtures pour faire entrer le bétail. » Avel Parra, 60 ans, agriculteur de La Toma, 2011.

44 Les conflits d'usage dans cette communauté peuvent s'expliquer par le nombre important de zones humides (plus de 80 selon un agriculteur) qui implique un travail de conservation et d'inspection considérable. Le débat est ainsi orienté ces dernières années vers une exclusion définitive des animaux du páramo.

45 Selon les agriculteurs qui adhèrent à l'idée de protection des zones humides, y compris avec l'aide du parc, le bétail est formé principalement de chevaux, généralement sans aucun intérêt productif ou pratique, devenant semi-sauvages du fait d'une absence totale des propriétaires. Lorsqu'il s'agit de bovins, ces derniers sont beaucoup trop maigres, faibles et sauvages pour accomplir les travaux agricoles tels que le labour. La pratique ancestrale du pâturage dans le páramo, et donc le droit du páramo qui y est associé, est désormais fortement remise en question par les agriculteurs.

46 «Enlevons les animaux et nous serons en train de protéger les 80 [lagunas] [...] non seulement ils mangent l'herbe, mais ils la piétinent aussi et l'eau s'enfonce. Parfois même, ils s'enfoncent et meurent là-bas et pourrissent et contaminent les sources [...]» Cesar Mesa, 49 ans, Agriculteur et membre d'ACAR, La Toma.

47 On peut donc se demander pourquoi et comment la communauté agricole locale, qui jusque dans les années 1990 ne faisait absolument pas le même diagnostic piétinement un problème pour l'approvisionnement en eau pour l'irrigation et pour l'eau potable des vallées -, en est venue à véhiculer ces nouvelles représentations du fonctionnement des zones humides et des interactions de ses pratiques avec ces zones humides. Ce diagnostic, commun aux principaux agents du parc et aux écologues, contribue de plus à l'adhésion de l'ensemble des acteurs à l'idée qu'il est nécessaire d'intervenir, ne pas laisser ces espaces à « l'abandon », tout particulièrement dans un objectif de protection quelles que soient les relations - écologiques, paysagères ou mystiques - alors même que ces espaces repoussaient et qu'il fallait que les pratiques anthropiques soient minimales auparavant comme nous l'avons vu précédemment.

48 "L'idée est venue d'aller voir là-bas pourquoi il n'y avait plus d'eau, quand nous sommes montés là-bas nous nous sommes rendus compte que les zones humides étaient sèches. Pourquoi ? Parce qu'il y avait beaucoup d'animaux pâturant, qu'il n'y avait pas de protection forestière sur ces sources, parce que le bétail avait consommé toute la diversité biologique qui est près des sources, près de l'origine des sources, ... logiquement, c'est une marque de désamour, d'oubli, de nous humains, envers cette mère, une évidence. C'est comme vous avec votre mère, vous partez en voyage, vous ne lui écrivez jamais, vous ne l'appelez jamais, alors elle se laisse aller à sa douleur et au désespoir jusqu’à ce quelle meure. [...] Chaque fois, un désamour de cette eau, cette terre, cet air. » Ligia Parra, Misintá, responsable de l'association ACAR, 2011.

Les enquêtes menées et l'observation effectuée ont permis d'identifier le rôle important de l'association ACAR et de la relation mystique qu'elle véhicule notamment au cours de rituels au sein du páramo (photo 2).

Photo 2 : Rituel autour de la laguna de Llano del Hato en mars 2012 (Blot, 2012) 

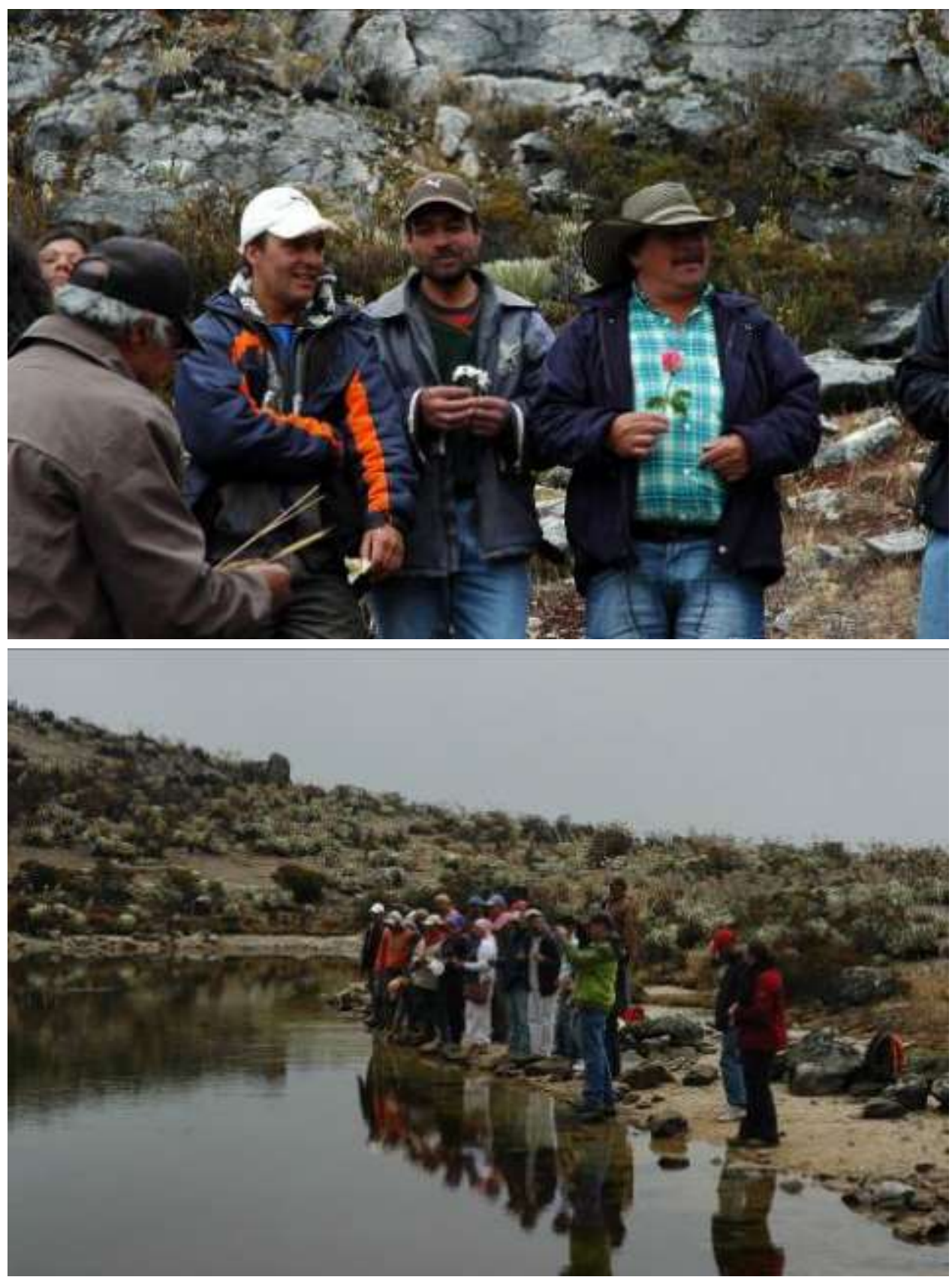

\section{Acar, médiateur?}

L'association ACAR réalise des inventaires, des suivis d'action de revégétalisation après dégradation par les animaux, procède aussi à la mise en place de clôtures de protection (photo 3). La protection concerne surtout la restriction d'accès au bétail qui altère le point d'émergence de l'eau par piétinement (mise en place de périmètres de protection par clôtures). Ses actions se focalisent aussi sur la restauration des cours d'eau (qui sont colonisés par la végétation en période de basses eaux, ces dernières étant accentuées par les prélèvements d'irrigation), mais aussi par la création de "viviers »- pépinières - de plantes autochtones pour la forestation des sources et de certaines berges de cours d'eau. Elle agit de concert avec les comités de riego, bien présents sur le terrain pour détecter des situations à problème et disposant de la main d'œuvre nécessaire pour intervenir sur le terrain. Elle assure, par ailleurs, un important volet pédagogique, puisqu'elle forme les agriculteurs.

\section{Photo 3 : ACAR en action :}



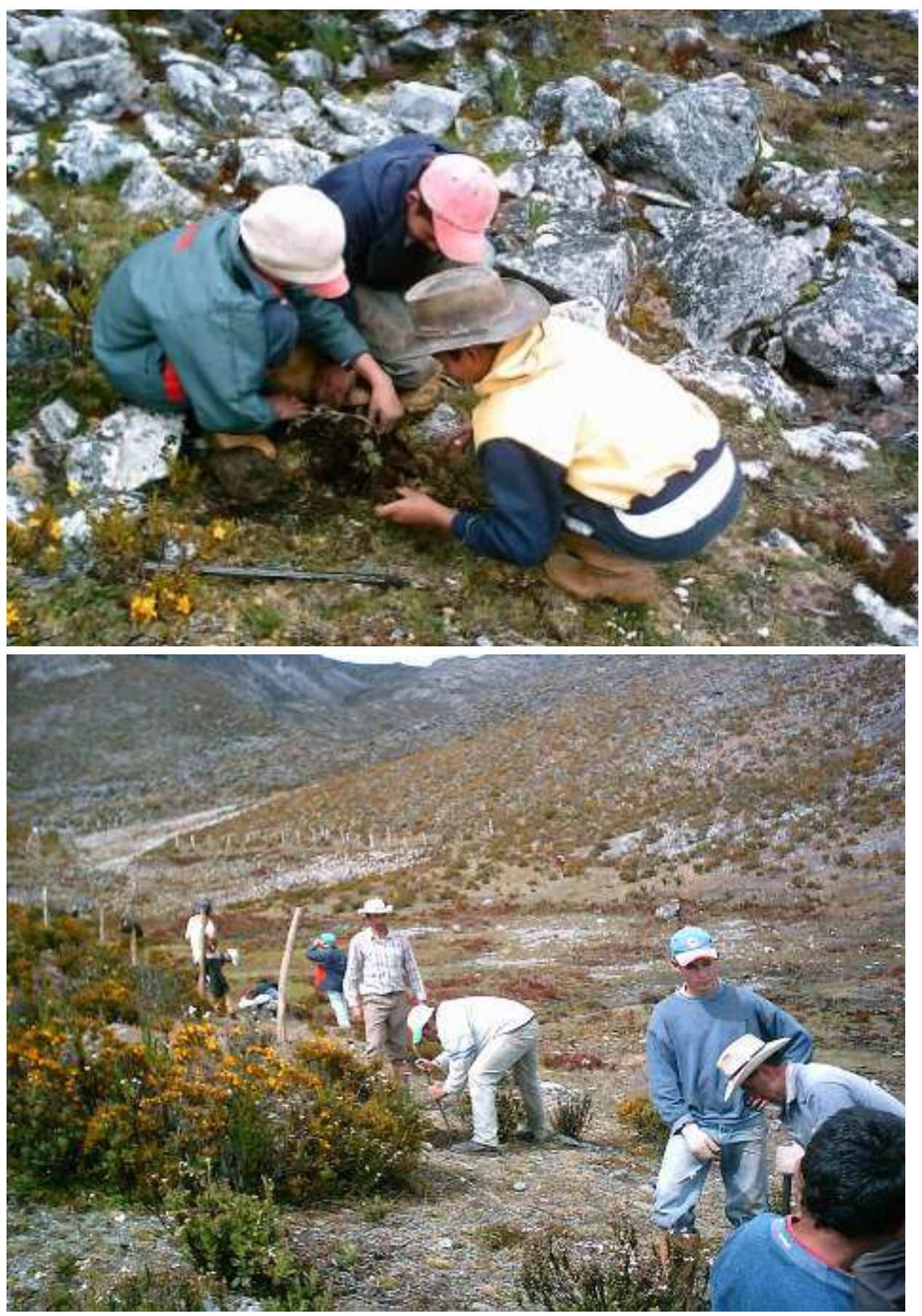

(a) Replantation de plantes autochtones issues du vivero (ACAR) (b) Travail collectif pour la mise en place d'un périmètre de protection (ACAR)

$51 \quad$ Les mesures de protection des zones humides développées par les agriculteurs via l'association ACAR sont approuvées par les représentants du parc (INPARQUES) ce qui se traduit concrètement par une assistance matérielle (fourniture de piquets par exemple) à l'association ACAR, mieux acceptée par les agriculteurs que l'intervention directe du parc.

Selon les agriculteurs, mais aussi selon les agents du parc et les écologues, les conséquences des pratiques de protection - notamment la réponse des milieux légitiment et justifient ces pratiques visant à la protection des zones humides : qu'elles soient d'ordre technique ou mystique. En effet, malgré les réticences initiales du monde agricole, les actions d'ACAR ont rapidement été acceptées et généralisées dans la plupart des comités de riego, notamment du fait de leurs " résultats spectaculaires », comme le souligne ce jeune agriculteur :

53 "Quand nous avons commencé avec les sources, personne n'y croyait, mais quand nous avons commencé à voir des résultats, que l'eau augmentait, nous avons commencé à nous intéresser à tout cela, pour que l'eau augmente. » Omero Rangel, 27 ans, agriculteur de Misintá, 2011. 
Les effets bénéfiques de ces mesures de protection sont aujourd'hui unanimement soulignés par les communautés de la vallée. Les représentations qu'ils se font du fonctionnement du milieu semblent confortées par l'impact qu'ils attibuent à leurs pratiques concrètes et mystiques.

" Après avoir clôturé toutes les sources, nous avons vu que l'herbe et les champignons commençaient à sortir, tout seuls. L'eau a commencé à augmenter, et cela était quelque chose de magique [...] en voyant que l'eau commençait à naître et que tout devenait plus vert, nous avons commencé à semer des plantes, les mêmes qu'il y avait juste à côté, nous avons commencé à les mettre à l'intérieur du parc. Comme cette source nous avait donné des résultats, nous avions compris que c'était cela que nous devions faire.» Ligia Parra, Misintá 2011.

"En haut, il y avait une source d'où sortait très peu d'eau. Nous l'avons fermé. Un mois après nous sommes montés, et la laguna était rempli à ras bord, on l'appelle "la laguna de la esperenza », ce fut merveilleux! » Jésus Rivera, 36 ans, agriculteur de Mocao, 2011.

Figure 4 : Construction convergente des relations aux zones humides des páramos andins de la Vallée du Haut Chama

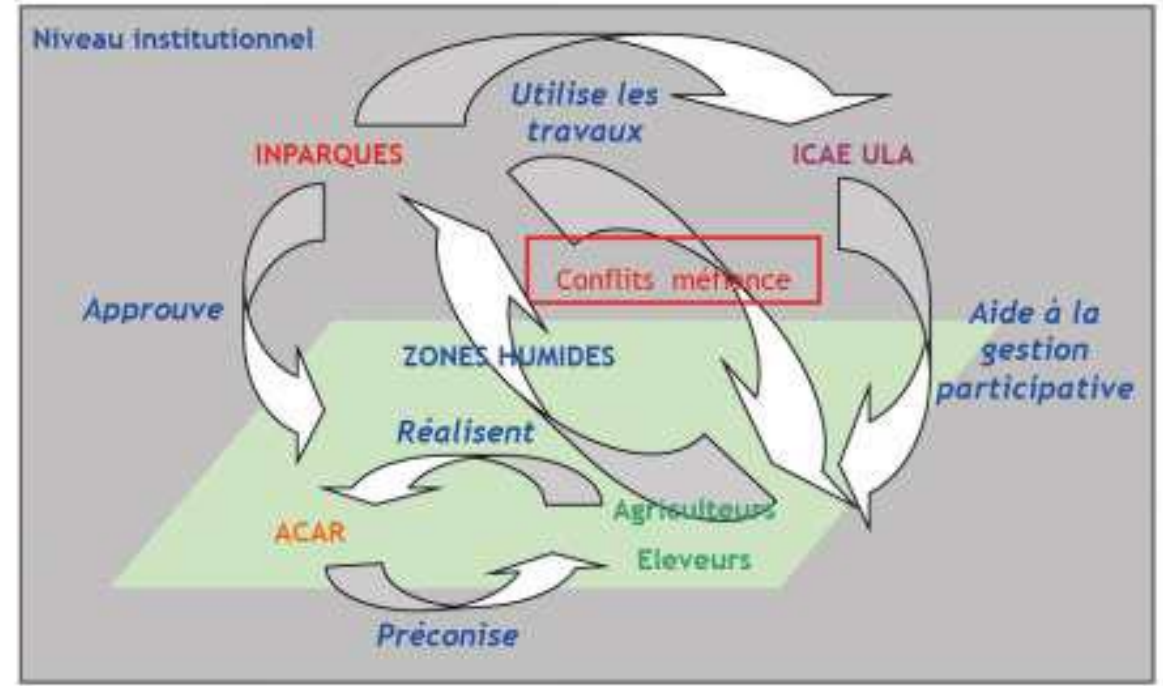

Les actions d'ACAR constituent même un facteur de cohésion social. En effet, les différentes tâches en lien avec la restauration des zones humides représentent une occasion pour que l'ensemble de la communauté se réunisse pendant une journée avec un objectif commun.

"Tout le monde y va, y compris les femmes et les enfants. On partage tout " Geraldo Romero, 42 ans, agriculteur de Mocao, 2011.

$\mathrm{Au}$-delà de la relation aux zones humides l'ambition d'ACAR est de ressusciter les rituels « ancestraux " - "nous sommes en train de réintégrer tout ce qui est lié à la magie, aux rites, que pratiquaient nos ancêtres aux éléments, comme nous disons, mais je crois que c'est un manque de respect de parler ainsi, car il s'agit de notre dieu qui donne la vie. L'eau est un dieu, la terre est un dieu, l'air, le sol ... C'est pour cela que nous enseignons à nouveau aux gens comment monter à la montagne pour la vénérer, pratiquer un rite sacré avec des fleurs, avec du miel, avec de l'encens, avec la prière, avec le rire et le chant ».

«En plus de sauver les sources, nous sauvons notre culture ancestrales. » Ligia Parra, 2012.

Cette opération de ré-enchantement des relations aux zones humides d'ACAR (croyances, chansons, mythes " ancestraux ") est très bien perçue par les agriculteurs :

"On n'avait pas à cette époque la vision pour conserver et entretenir, pour mettre sous clôture ces endroits. Nous devions aussi faire un travail spirituel, on devait apprécier l'eau, le Saint Isidro qui est le saint des agriculteurs. Nous allons là-bas, nous chantons, nous prions. » Dionsio Espinoza, 65 ans, agriculteur de Misintá, 2011. 
On a donc également une validation mystique des pratiques de l'ACAR. Espace délaissé pendant de nombreuses années, le páramo, et plus particulièrement ses zones humides, constitue donc un espace réapproprié par les communautés rurales. Beaucoup d'agriculteurs se disent désormais sensibles et attentifs à la bonne santé de ces espaces fragiles. Il est important de souligner que l'eau provenant des zones humides constitue également l'unique source d'eau potable pour ces communautés. Ainsi, nos entretiens font état d'une très forte implication des agriculteurs afin de s'assurer qu'aucun agriculteur ne sème prés de ces sources, ou pour vérifier si elles ne sont pas contaminées par des animaux morts. Les agriculteurs n'hésitent d'ailleurs pas à afficher leur grande fierté quant à la qualité de cette ressource, qui serait « cristalline comme de l'eau minérale, meilleure que de l'eau minérale, car l'eau minérale contient des produits chimiques alors que cette eau est complètement naturelle! » Felipe Torres, 40 ans, agriculteur de Mocao, 2011.

Il est important de souligner que cette relation mystique a été confortée par des épisodes pluvieux et une recharge des réseaux hydrographiques dès les années suivant les premières cérémonies. Selon les agriculteurs, ces évènements constituent la réponse positive de la «madre naturaleza » à leurs pratiques. Bien que très sceptiques au début du processus - pour eux Ligia Parra était une sorte de " hippie » qui après avoir parcouru le monde revenait au pays imposer sa vision du monde illuminée -, ils s'associent donc, depuis, à ces cérémonies. A présent, " las comunidades saben que es lo que tienen que hacer » - les communautés savent que c'est ce qu'elles doivent faire -, à la fois en termes de pratiques concrètes de protection physique des zones humides, mais aussi de pratiques spirituelles sous la forme de rituels cérémoniaux.

Bien que les pratiques anciennes soient de ne pas parcourir ces espaces, ou de le faire sans se faire remarquer, sans bruit, Ligia Parra contribue à construire un nouvel imaginaire mystique associé à une nouvelle vision des zones humides : avant, elles représentaient plutôt des risques et, aujourd'hui, des ressources. Une enquête approfondie sur l'histoire de ce personnage mérite d'être conduite pour identifier les éléments constitutifs de son habitus qui ont contribué à forger les représentations qui sont les siennes : vision systémique du fonctionnement des bassins-versants, connaissance des caractéristiques hydrologiques des milieux - milieux à forte capacité de rétention d'eau etc... ; mais aussi les principes rationnels qui président aux pratiques qu'elle incite - reforestation, protection des sources ou agriculture écologique.

Ce type de relations aux milieux pourraient, à l'aube du xxıe s., être remis en question par les connaissances produites par la communauté scientifique sur les milieux, à la fois en termes de qualification - identification de caractéristiques et de modes de fonctionnement, mais aussi en termes de pratiques - protections des zones humides, forestation... - ainsi que par les pratiques des gestionnaires de ces espaces comme le parc, mais ce n'est absolument pas le cas. Il apparaît même que la dimension mystique de la relation conforte pour un temps les nouvelles relations aux zones humides dans le páramo vénézuélien.

66 Mais les recommandations d'ACAR ne sont pas acceptées par toutes les communautés agricoles. Elles semblent plus facilement applicables à l'échelle des plus petits bassins versants, qui sont très sensibles aux variations hydrologiques - manque d'eau -, elles deviennent particulièrement difficiles à mettre en place dans des zones beaucoup plus étendues, comme La Toma, qui de surcroit subissent moins le manque d'eau. Il ne faut pas occulter les rapports de force qui opèrent entre agriculteurs au sujet de l'appropriation de ces espaces, traduite dans les représentations et les pratiques de protection. En effet, leur appropriation est directement liée à la maîtrise de l'eau, essentielle à la dynamique agricole actuelle. De plus, il faut souligner que la concordance d'années plus pluvieuses suite aux changements de pratiques et à la réalisation de cérémonies d'offrandes à la « madre naturaleza » a contribué à valider et à conforter l'engagement initié par ACAR de façon bien plus efficace que les recommandations des techniciens du parc. On pourrait même dire que le fonctionnement du milieu interagit dans la dynamique relationnelle : le manque d'eau de la fin des années 1990 a poussé les sociétés locales à s'interroger et à observer les milieux ; l'augmentation des pluies suite aux cérémonies d'offrande à la « madre 
naturaleza » conforte ce type de pratiques d'après les agriculteurs... mais aussi les chercheurs.

$67 \quad$ La question de la préservation des zones humides est donc relativement complexe. Elle met en scène une diversité d'acteurs au sein des communautés qui n'ont pas forcément les mêmes intérêts. Il semble toutefois qu'une grande majorité d'agriculteurs soit désormais favorable aux différentes mesures de protection que préconise ACAR ; en témoigne l'admiration, le respect et la sympathie de l'ensemble des agriculteurs à l'égard de sa coordinatrice, Ligia Parra.

\section{Conclusion}

68 Les zones humides du páramo ont, jusque dans les années 1970, représenté davantage de risques que de ressources pour les communautés agricoles andines du Venezuela. Aujourd'hui, on souligne leurs caractéristiques écologiques - biodiversité -, hydrologiques - zones tampons, réservoirs -, paysagères, ou encore mystiques. Ainsi, les zones humides du páramo vénézuélien font l'objet de multiples représentations. Bien que ces dernières divergent selon les types d'acteurs (agriculteurs, parc national, écologues, association de protection), leur rôle de réservoir hydrologique est reconnu par tous.

69 L'étude conduite montre que l'on assiste bien à une dynamique des pratiques et des représentations qui tend vers des principes de conservation des zones humides de façon dominante. La majorité des acteurs accorde de l'importance à ces zones humides et est entrée dans une logique de protection de ces zones, mais pour des raisons différentes : source d'eau/biodiversité. Ainsi, les intérêts convergent entre acteurs qui, jusqu'à il y a encore peu de temps (fin des années 1990), s'opposaient - protection de la biodiversité, préservation des capacités de rétention d'eau. Cette dynamique repose sur la coexistence de relations différentes, mais convergentes aux zones humides du páramo. Pourtant ces principes de conservation interrogent les pratiques existantes et, notamment, le pâturage de haute altitude dans le páramo vénézuélien. La convergence d'intérêts entre les chercheurs, le parc et les agriculteurs regroupés au sein de l'association ACAR se révèle être efficace pour préserver du piétinement.

Dans ce processus, la construction de nouvelles représentations - hydrosystémiques - et l'adhésion à une logique de protection des communautés rurales ont été fondamentales. Elles sont avant tout l'œuvre d'une association de protection de l'eau, ACAR. Croisant pratiques mystiques et actions fonctionnelles de préservation, ACAR a construit de nouveaux savoirs sur ces espaces. De fait, ses incitations pratiques se révèlent être bien plus efficaces que les restrictions d'usages imposées par le parc national et marquées par des conflits avec les communautés. En termes de relations aux zones humides, bien que les agriculteurs reconnaissent les zones humides comme une composante naturelle d'importance, il semble que leurs actions de préservation soient avant tout motivées par le bon fonctionnement hydrologique de ces espaces, pourvoyeurs d'eau pour les zones aval (relation fonctionnaliste).

71 L'adhésion de l'ensemble des communautés agricoles à ce type de relations demeure l'objectif des gestionnaires du parc. Selon eux, une classification des zones humides en tant que site RAMSAR pourrait - parce qu'associée au principe de participation -, dans un futur proche, permettre de mieux travailler avec les communautés locales.

"Quand nous créons un site RAMSAR, on créé alors un comité RAMSAR et qui est composé par les membres de la communauté qui s'y trouve. Cela nous permettrait d'avoir plus de proximité pour le développement de certains projets de conservation et d'utilisation durable de ces espaces. » Ingénieur 1 INPARQUES, 2011.

73 L'intégration de ces zones humides dans la convention RAMSAR est en attente depuis 2007. Elle pourrait permettre d'établir des programmes d'actions spécifiques pour les zones humides alto andines et les bassins qui les alimentent, afin de préserver leur biodiversité, leurs fonctions régulatrices vis à vis des flux d'eau et comme espace de vie de beaucoup de communautés locales, programmes en adéquation avec les représentations des zones humides du parc. Depuis décembre 2013, il existe de plus un 
projet de création de réserve de biosphère dans les páramos du Venezuela. Cette réserve de biosphère, si elle est validée, serait une reconnaissance par l'UNESCO de ces espaces comme zones modèles conciliant la conservation de la biodiversité et le développement durable, dans le cadre du Programme sur l'homme et la biosphère (Man And Biosphere, MAB).

\section{Bibliography}

ANGÉLIAUME-DESCAMPS A., 2010, Acteurs locaux et gestion participative de la ressource en eau dans un contexte d'activité productive intensive dans le Parc national de la Sierra Nevada (Venezuela), in MICOUD A. (éd.), Espaces protégés : acceptation sociale et conflits environnementaux, Collection Edytem, nº 10, p. 25-39.

ANGÉLIAUME-DESCAMPS A., 2012, Les zones humides des páramo des hautes vallées des Andes vénézuéliennes : d'une gestion collective à une gestion participative de la ressource en eau, Colloque annuel du GHZH, Usages et espaces communautaires dans les zones humides, Thème 1 : Exploitation, partage des ressources et conflits, 12 et 13 novembre $2010 \mathrm{GHZH}-$ Orléans, Publication Revue du GHZH, p. 11-24, 135 p.

ANGÉLIAUME-DESCAMPS A., BLOT F., MOLINA L., LEROY D., MAIRE E. PELTIER A., ANTOINE J. M., 2013, Construction des ressources en eau au sein d'un espace agricole et protégé de montagne tropicale : Les facteurs de la mise en place d'une gestion participative de la ressource en eau au sein du Parc National de la Sierra Nevada - Venezuela, in CHARLERY de la MASSELIĖRE B., THIBAUD B., DUVAT V. (dir.), Actes du colloque des Journées de Géographie Tropicales, PUM, Toulouse, 16-19 Mars 2011, Les territorialités rurales des sud en question, atelier 3 : Territorialités et construction des systèmes de ressources, Dynamiques rurales dans les pays du Sud, l'enjeu territorial, mai, p. 207-226, 414 p.

BECK C. et al. (dir.), 2007, Les Zones humides européennes : espaces productifs d'hier et d'aujourd'hui. Actes du premier colloque international du Groupe d'Histoire des Zones Humides, Le Blanc, 21-23 octobre 2005, Aestuaria. Culture et développement durable, Collection Histoire et terres humides, Cordemais, Estuarium, $515 \mathrm{p}$.

BECK C. et al., 2006, Temps et espaces des crises de l'environnement, Versailles, Quæ Indisciplines, $416 \mathrm{p}$.

BLOT F., 2005, Discours et pratiques autour du « développement durable » et des « ressources en eau ». Une approche relationnelle appliquée aux bassins d'Adour-Garonne et Segura, Thèse de doctorat, Université de Toulouse Le Mirail, 542 p.

BURNOUF J. et al. (dir.), 2004, Fleuves et marais, une histoire au croisement de la nature et de la culture, Paris, CTHS, $493 \mathrm{p}$.

DE ROBERT P., 2001, Apprivoiser la montagne, portrait d'une société paysanne dans les Andes (Venezuela), IRD éditions, $417 \mathrm{p}$.

INPARQUES, 2007, Complejo de Humedales Cuenca Alta del Río Chama - Sistema Lagunar Santo Cristo Parques Nacionales Sierra Nevada y Sierra de La Culata, 18 p.

LLAMBÍ L.D., FARIÑAS M., SMITH J.K., CASTAÑEDA S.M., BRICEÑO B, 2013, Diversidad de la vegetación en dos páramos de Venezuela : un enfoque multiescalar con fines de conservación, in CUESTA F., SEVINK J., LLAMBÍ L.D., MALDONAD G., DE BIEVRE B (Eds.), Investigación para la Conservación en dos Páramos Andinos. Proyecto Páramo Andino (PNUMA- GEFCONDESAN), Quito, Ecuador.

LLAMBÍ L.D., SARMIENTO L., RADA F., 2013, La evolución de la investigación ecológica en los páramos de Venezuela : múltiples visiones de un ecosistema único, in MEDINA E., HUBER O., NASSAR J.M., NAVARRO P. (Eds.), Recorriendo el paisaje vegetal de Venezuela. Ediciones IVIC, Instituto Venezolano de Investigaciones Científicas (IVIC), Caracas, Venezuela, p. 173-209

LERMIS A. LARA P., 2007, Validación del instrumento para la caracterización e inventario de humedales altoandinos, sector Micarache, subcuenca de Gavidia, Parque National Sierra Nevada, Estado Merida, Venezuela, Trabajo de grado presentado ante la Universidad de los Andes como requisito parcial para optaral título de Ingeniero Forestal, 96 p.

LUGINBHUL Y., 1992, Nature, paysage, environnement, obscurs objets du désir de totalité, in ROBIC M.-C. (dir.), Du milieu à l'environnement. Pratiques et représentations du rapport homme-nature depuis la renaissance, Paris, Economica, p. 11-56.

MOLINILLO M., MONASTERIO M., 2005, El Complejo de Humedales del Páramo El Blanco, in Turberas Altoandinas : Espacios frágiles de vida y cultura, Quito, Ecuador, en Izurieta p. 7-15.

MONASTERIO M. (ed.), 1980, Estudios ecológicos en los páramos andinos, Ediciones de la Universidad de los Andes, Mérida, Venezuela.

MONASTERIO M., MOLINILLO M., 2003, La integración del Desarrollo agrícola y la conservación de áreas frágiles en los páramos de la Cordillera de Mérida, Venezuela, Congreso Mundial de páramos, Tomo II. (Paipa, 2002), p. 734-749. 
OUZOUF-MARIGNIER M.-V., 1992, L'environnement vu par les notables locaux à la fin du xviiiè siècle, in ROBIC M.-C. (dir.), Du milieu à l'environnement. Pratiques et représentations du rapport homme-nature depuis la renaissance, Paris, Economica, p. 57-88.

POINT P., 2010, Quelle méthodologie pour la confrontation des valeurs de conversion ou de conservation des zones humides ?, Revue d'Économie Régionale et Urbaine, $\mathrm{n}^{\circ} 5$ décembre, p. $855-876$. DOI : 10.3917/reru.105.0855

DOI : $10.3917 /$ reru.105.0855

RAFFESTIN C., 1980, Pour une géographie du pouvoir, Paris, LITEC, 249 p.

RODRÍGUEZ M., 2010, El páramo como proveedor de agua : análisis de las unidades geomorfológicas y de vegetación sobre el balance hídrico de una microcuenca andina de Venezuela. Microcuenca alta de la Quebrada Miguaguó-Mixteque. Postgrado en Ecología Tropical, Universidad de los andes, ICAE, $140 \mathrm{p}$.

SALLES J.-M., 2010, Évaluer la biodiversité et les services écosystémiques : pourquoi, comment et avec quels résultats ?, Natures Sciences Sociétés, Vol. 18, nº 4, p. 414-423.

TORO J., GARCÍA A., ROMERO L., 2008, Efectos del calentamiento global ¿̇ Nieves eternas en la Sierra Nevada de Mérida ? Investigación, $n^{\circ}$ 17janvier, p. 90-93.

VALERO LACRUZ L., 2010, Efecto de la exclusión del pastoreo sobre humedales alto andinos en la Sierra Nevada de Mérida, Maestría, ULA, 152 p.

VARGAS RIOS O., VELASCO-LINARES P., 2011, Reviviendo nuestros Páramos, Restauración ecológica de páramos, Proyecto Páramo Andino, 183 p.

\section{Notes}

1 Nous considérons les relations aux zones humides, articulation des représentations et des pratiques des individus et groupes à l'égard de zones humides, comme la résultante des processus de régulation qui président à leur inter-construction (Blot, 2005, p. 25-28).

$2 \mathrm{Au}$ sens des écologues, milieu de haute montagne en zone tropicale, écosystèmes typiques des Andes septentrionales, qui désigne un sorte de lande d'altitude, correspond à la partie haute de l'étage andin $2000 \mathrm{~m}$. $-4000 \mathrm{~m}$. et à l'étage alti-andin 4 000-5000 m., hot-spot de biodiversité (Vargas Ríos, Velasco-Linares, 2011, p. 17). Pour les populations rurales des Andes vénézuéliennes, terres d'en haut, se définit en opposition aux terres d'en bas cultivées ou cultivables (De Robert, 2001, p. 217) zone d'altitude, lieu marginal. Depuis plusieurs siècles cet espace est soumis à un usage restreint en raison du froid qui y règne : espace de pâturage, consacré à l'élevage extensif, ou espace de passage de la vallée centrale vers les basses terres, ou occasionnellement lieu pour cueillir des plantes médicinales.

3 ICAE : Instituto de Ciencias Ambientales de l'ULA, Universidad de Los Andes.

4 INPARQUES : Instituto Nacional de PARQUES, PNSC et PNSN, Parque Nacional de la Sierra Culata et Parque Nacional de la Sierra Nevada, correspondant aux deux sierras de part et d'autre du sillon central inter-andin.

5 ACAR : Asociacion de Coordinadores del Ambiente por los agricultores del Municipio Rangel.

6 Les zones humides du páramo andin sont essentiellement associées aux lacs glaciaires, lagunas. Ces lacs font, en fait, partie d'un cortège beaucoup plus vaste de zones humides : tourbières (turbera de musgo), marais (pantanos) et pelouses humides (céspedes). Ces zones marécageuses sont disposées, en générale, dans le courant qui provient ou alimente les lagunas, à un niveau altitudinal compris entre 3600 et $4200 \mathrm{~m}$. (Monasterio, Molinillo, 2003). Ces zones humides sont liées tant aux caractéristiques climatiques que morphologiques du páramo. Elles ne représentent qu'un peu moins de $10 \%$ de la superficie du páramo (Molinillo, Monasterio, 2005), soit près de 3000 hectares pour les marais et pelouses humides et 300 hectares pour les lagunas dans le haut bassin versant du Chama, secteur de notre étude (INPARQUES, 2007).

7 Travaux développés dans le cadre d'un projet PICS CNRS INEE « Mutations des montagnes tropicales et environnement, Vulnérabilité liée à l'eau dans les Andes vénézuéliennes »20102012.

8 Lac d'origine glaciaire.

9 "Derecho de páramo », droit accordé pour le pâturage de leurs animaux aux indigènes par les colons espagnols qui s'étaient approprié les terres agricoles basses consacrées, entre autre, à la culture du blé.

10 Lorsque les Espagnols occupent cette région dès le XVe s., c'est pour la niche agroécologique qu'elle constitue. Ils introduisent alors dans le cadre des grandes encomiendas la culture du blé que les colons ne pouvaient importer d'Espagne - et l'usage de l'araire ou encore des bovins, tout en conservant certaines productions et pratiques traditionnelles.

11 L'étage tempéré de cette montagne tropicale constitue une niche agroécologique (condition d'ensoleillement du tropique avec des températures tempérées) mais aussi fenêtre écologique (absence de saison thermique et influence du climat tropicale de montagne). L'irrigation, en 
permettant de produire toute l'année et d'assurer jusqu'à 4 récoltes, devient une condition essentielle de réussite agricole.

12 En 1952, le PNSN se met en place, avec un zonage particulièrement restrictif : sur le versant nord, terrain de l'étude, les très hautes terres sont classées en ZPI (toutes activités interdites, statut conservationniste) ou en ZPS (autorisation des activités de recherches et quelques rares activités touristiques, statut protectionniste) et de tous petits espaces sont accordés à l'agriculture (ZA). Dans ce contexte, le parc donne une nouvelle valeur à l'eau (et aux zones humides) : une valeur écologique, l'objectif premier du parc étant de préserver les écosystèmes de montagne. Dans un premier temps, il exerce une pression forte sur les populations puisqu'il se pose en fournisseur de service environnemental et entend ainsi protéger les zones humides et la ressource en eau pour des usages agricoles très localisés.

13 Une analyse de la structure de la végétation dans le petit bassin versant de Gavidia, proche de la zone d'étude, montre que les zones humides ne couvrent que $6 \%$ du bassin versant, mais que $70 \%$ des espèces identifiées ne sont présentent dans aucune autre formation végétale du bassin versant (Llambí, Smith et Escalona, données non publiées, in INPARQUES, 2007).

14 Depuis quelques années, cet institut travaille en contact permanent avec les communautés, afin de les orienter vers une meilleure gestion des espaces fragiles. À ce titre, de 2007 à 2012, ICAE a coordonné, au Venezuela, le projet de conservation de la biodiversité du páramo dans les Andes du nord et centrale, mieux connu comme « le Projet Páramo Andino » (PPA). L'objectif principal du PPA était de trouver un compromis entre les activités des communautés qui vivent dans le páramo (Équateur, Pérou, Colombie et Venezuela) et la conservation de cet écosystème (Llambí, Sarmiento et Rada, 2013).

15 Torrents de montagnes, ex. Quebrada El Royal.

16 Grandes rivières de montagne, ex. Río Chama.

17 ACAR : Asociacion de Coordinadores de Ambiente por los agricultores del Municipio Rangel, réalise des inventaires, des états des lieux, des suivis d'action de revégétalisation après dégradation par animaux, de mise en place de clôtures protection. Les membres d'ACAR sont pour la plupart des agriculteurs.

18 Les comités de riego sont les structures collectives de gestión de l'eau d'irrigation, gérées par les agriculteurs; elles sont apparues dans les années 1970 à la suite des premiers programmes de développement agricole.

19 Diversité des termes locaux utilisés pour caractériser les diverses zones humides, de fond de vallées, de bas de versants, de bord de lagunas... (Angéliaume-Descamps, 2012).

20 Les écologues soulignent qu'au-delà de la diminution de la biomasse ils notent surtout une réduction notable de la richesse des espèces dans les parcelles pâturées : certaines se voient favorisées par le pâturage (e.g. Aciachne pulvinata, Espeletia schultzii), alors que d'autres sont affectées négativement (e.g. Trisetum irazuense) (ICAE 2004, non publié in Lermis, 2007).

\section{List of illustrations}

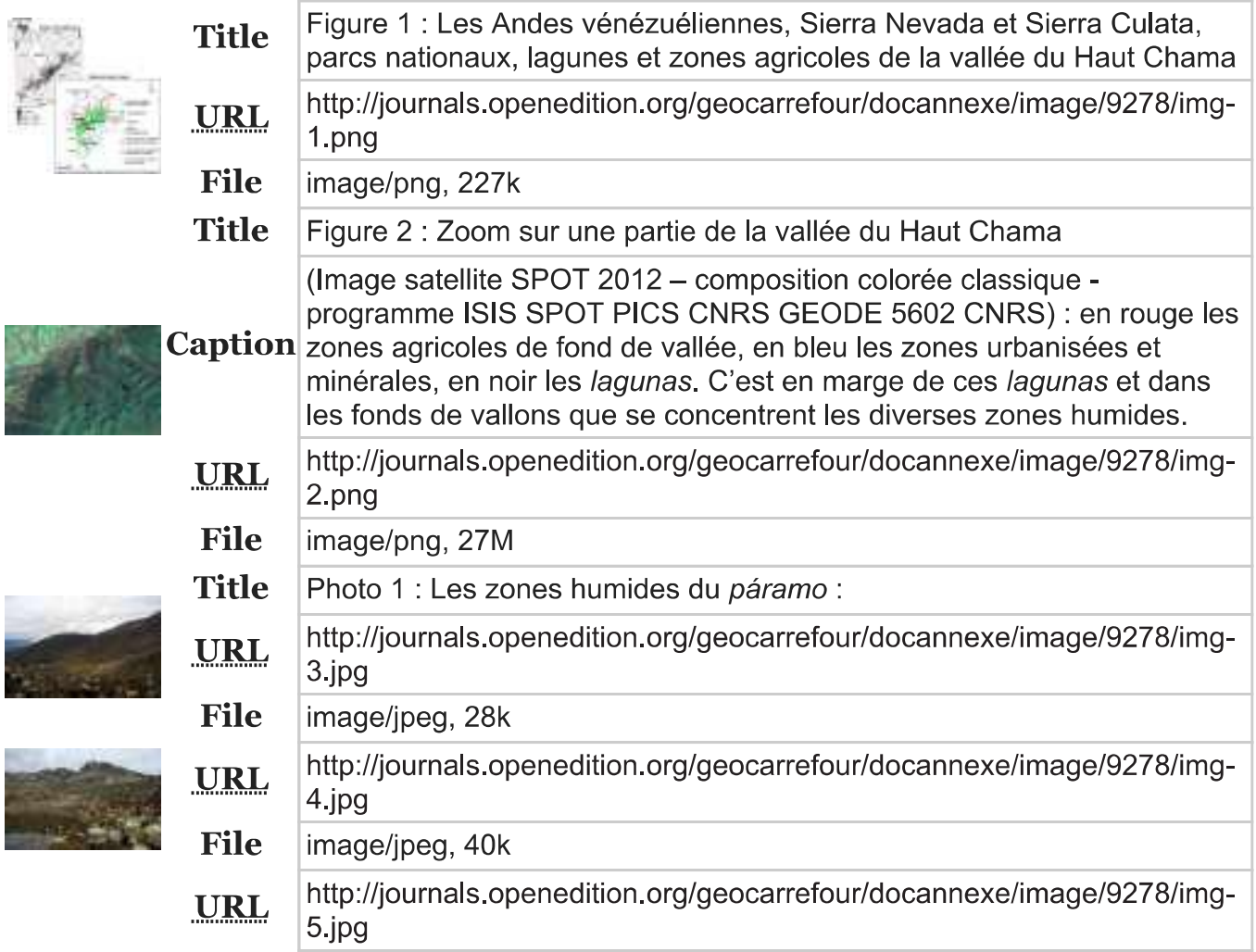




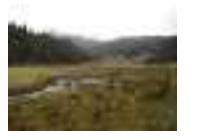

File image/jpeg, 28k

a) zones humides à l'amont de Llano de Hato, Sierra Culata (Blot, 2012) (b) lagunas de Llano de Hato, Sierra Culata (Blot, 2012), (c) amont de la

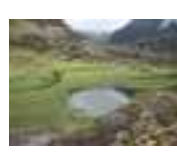

Caption

fond de vallée et prise d'eau du périmètre irrigué de Mocao, Sierra

Nevada (Angéliaume, 2010)

URL

http://journals.openedition.org/geocarrefour/docannexe/image/9278/img6.jpg

File image/jpeg, 44k

Title

Figure 3 : Evolution temporelle des représentations et des enjeux des

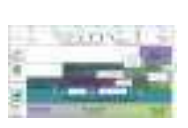

URI vallée du Haut Chama

File image/png, 375k

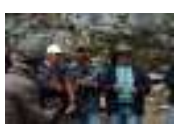

Title

Photo 2 : Rituel autour de la laguna de Llano del Hato en mars 2012 (Blot, 2012)

URL

http://journals.openedition.org/geocarrefour/docannexe/image/9278/img8.jpg

File image/jpeg, 40k

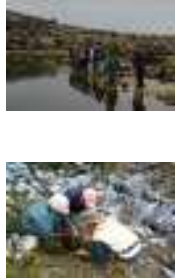

URL

http://journals.openedition.org/geocarrefour/docannexe/image/9278/img-

File 9.jpg

Title Photo 3 : ACAR en action :

URL http://journals.openedition.org/geocarrefour/docannexe/image/9278/img10.jpg

File image/jpeg, 56k

(a) Replantation de plantes autochtones issues du vivero (ACAR) (b)

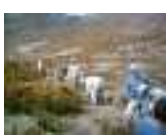

Caption Travail collectif pour la mise en place d'un périmètre de protection (ACAR)

URL http://journals.openedition.org/geocarrefour/docannexe/image/9278/img11.jpg

File image/jpeg, 48k

Title

Figure 4 : Construction convergente des relations aux zones humides des páramos andins de la Vallée du Haut Chama

URI http://journals.openedition.org/geocarrefour/docannexe/image/9278/img12.png

File image/png, 69k

\section{References}

Bibliographical reference

Alexandra Angéliaume-Descamps, Frédérique Blot and David Leroy, « Dynamique récente des relations aux zones humides des páramos andins vénézuéliens : entre fonctionnalisme et mystique », Géocarrefour, vol. 88/4 | 2013, 285-298.

Electronic reference

Alexandra Angéliaume-Descamps, Frédérique Blot and David Leroy, « Dynamique récente des relations aux zones humides des páramos andins vénézuéliens : entre fonctionnalisme et mystique », Géocarrefour [Online], vol. 88/4 | 2013, Online since , connection on 09 April 2018. URL : http://journals.openedition.org/geocarrefour/9278 ; DOI : 10.4000/geocarrefour.9278

\section{About the authors}

Alexandra Angéliaume-Descamps

GEODE UTM 5 Allées Antonio Machado 31058 Toulouse angeliau@univ-tlse2.fr 
David Leroy

GEODE UTM 5 Allées Antonio Machado 31058 Toulouse david4leroy@hotmail.fr

\section{Copyright}

(C) Géocarrefour 